\title{
VICTOR HUGO ET LA RÉVOLUTION INDUSTRIELLE : QUAND UN ROMANTIQUE SE FAIT APÔTRE ET JUGE DE LA SCIENCE
}

\section{VICTOR HUGO AND THE INDUSTRIAL REVOLUTION: WHEN A ROMANTIC IS APOSTLE AND JUDGE OF SCIENCE}

\author{
Moustapha Faye \\ Université Gaston Berger de Saint-Louis du Sénégal, Sanar, Senegal
}

Résumé : On peut facilement remarquer, à travers l'histoire, le rapport plus ou moins direct que les écrivains entretiennent avec les grandes crises sociales ou les révolutions. Parfois ils y prennent directement part, généralement en tant que instigateurs (on sait ce que la Révolution française de 1789 doit aux écrits politiques d'un Rousseau et d'un Voltaire) ; d'autres fois ils s'en constituent en juges, postfaçant ce qu'ils n'ont pas décidé. En marge de ces deux catégories, on peut en trouver une troisième : des auteurs qui, en même temps que juges, deviennent des témoins (passifs ou actifs) des événements. Victor Hugo appartient à ce dernier lot. Contre toute attente, la figure la plus emblématique du romantisme français place la science au cœur de son œuvre. Grand auteur du XIX siècle, il est contemporain d'une Révolution industrielle qu'il appelle d'abord de tous ses vœux avant que sa déception s'attelle à en montrer tout le mal pour l'humanité.

Mot clés: Machinisme; Misère ; Progrès ; Révolution ; Roman.

Abstract: Through history, we can easily notice the more and less direct relationship which writers keep up with big social crises or revolutions. Sometimes, they take a direct stand in them: this is the case in which they become the very initiators of such events (as it has been said, the French Revolution took the cue, to some degree, from Rousseau's and Voltaire's writings). Other times they can stand as judges, writing by the way an epilogue on what they did not decide. Beyond these two categories, there is a third one - authors who constitute themselves as witnesses (passive or active) of the events at the same time that they stand as judges. Victor Hugo belongs to this last group. Against all expectations, the most emblematic figure of French Romanticism places science at the very core of his work. Great writer of the 19th century, he is cotemporary of an Industrial Revolution whose development he praises and wishes for, before a great disappointment leads him to unveil and show all the evil effects for mankind.

Keywords: Machinery; Misery; Novel; Progress; Revolution.

Le lien entre les écrivains et les grandes révolutions humaines est une évidence que certains analystes expliquent par la dialectique pensée-action. Par exemple, parlant du cordon ombilical reliant la philosophie des Lumières à la Révolution française, Alphonse Dupront écrit : «Ce qui s'était préparé 
par le remue-ménage des esprits recevait sa consécration sur le théâtre de la politique. Ce que la première avait discrédité, la seconde l'abolit. Ce que la première avait imaginé, la seconde l'instaure " (DUPRONT, 1996, p. IV). Par ailleurs, quand l'esprit trop pointilleux des analystes refuse de voir cette dite évidence, certains auteurs ne se font pas faute d'établir eux-mêmes les corrélations. Il en est ainsi de Victor Hugo lors du coup d'État de Napoléon III, le 2 décembre 1851. Daniel Bougnoux assurait que "Hugo n'a pas cherché le 2 décembre 1851 mais il s'est montré extrêmement réactif à l'événement : le coup d'État en fit l'homme d'un parti, c'est-à-dire poète qui subordonne son œuvre à l'action" (BOUGNOUX, 2002, p. 56).

Le thème du progrès, chez un écrivain du XIX ${ }^{e}$ siècle, ne peut en aucun cas surprendre un lecteur, le siècle étant celui de la Révolution industrielle ${ }^{1}$. Georges Bafaro explique :

$\mathrm{Au} \mathrm{XIX}^{\mathrm{e}}$ siècle, les découvertes scientifiques se multiplient ainsi que les applications pratiques qui en sont tirées. L'essor des méthodes mises en œuvre et leur fécondité ouvrent une ère nouvelle, placée sous le signe de la machine, indéfiniment perfectible, et marquée par la conviction que les secrets de l'univers finiront un jour par être percés. La matérialisation rapide des avancées théoriques transforme continument la vie quotidienne. Le constat réjoui ou inquiet d'un changement rapide de la face du monde influence les mentalités jusque dans le domaine artistique. (BAFARO, 1995, p. 15)

Pourtant Hugo se faisant héraut du progrès peut déconcerter. L'un des dangers les plus récurrents de la périodisation en littérature c'est la réduction d'envergure qui en accompagne le principe. Ainsi Victor Hugo le plus souvent est définitivement rivé à la statue scolaire de chef de file du romantisme littéraire français au XIX ${ }^{e}$ siècle, courant littéraire dont les thèmes fondamentaux sont la communion avec mère nature, le rêve, l'amour, le moi, etc., autrement dit les dignes contraires de l'esprit scientifique. Pourtant, en ce qui concerne Victor Hugo, il ne faut point perdre de vue qu'il est trop homme de son temps pour se soustraire à un seul de ses mouvements d'envergure. Qu'on garde aussi à l'esprit cette réalité propre au XIX ${ }^{\mathrm{e}}$ :

\footnotetext{
${ }^{1}$ Jauss faisait remarquer en ce sens les limites des thèses formalistes par trop centrées sur l'autonomie du fait littéraire : "L'historicité de la littérature ne se réduit pas à la succession des systèmes de formes et des esthétiques; comme l'évolution de la langue, celle de la littérature se définit non seulement par l'intérieur, par le rapport spécifique qu'entretiennent en elle la diachronie et la synchronie, mais aussi par son rapport avec le processus général de l'histoire " (JAUSS, 2015, p. 47).
} 
Ce siècle dans son ensemble consacre en France et en Europe grâce à la révolution industrielle le triomphe sans précédent de la science élevée désormais par le positivisme au rang de pierre angulaire d'une religion laïque de l'humanité. Auguste Comte apparaît comme le principal théoricien et parle de phase scientifique ou état positif. Ses principes vont imprégner tous les autres domaines de connaissance. Il n'est pas jusqu'à la littérature qui n'ait une affiliation avec la science. Le poète - Hugo surtout - se fait le chantre du progrès avec l'incarnation de la figure prométhéenne chargée de conduire le peuple et d'illuminer l'avenir. (BAFARO, 1995 p. 21 [nous soulignons, $\mathrm{MF}]$ )

C'est - entre autres considérations - ce rapport particulier du romancier à la science qui pousse André Maurois à le sacrer « le maître des mots français ». Dans la philosophie de cet académicien, la nécessité pour un auteur moderne de s'approprier les connaissances scientifiques de son temps s'inscrit dans le programme d'un nouvel art poétique :

Comment comprendriez-vous le monde moderne si vous en effacez, par ignorance, ce qui est son travail et son orgueil: la recherche scientifique? Aldous Huxley soutenait qu'il est inadmissible qu'un homme cultivé croie devoir connaître une œuvre de Shakespeare, mais non la deuxième loi de la thermodynamique. (MAUROIS, 1966, p. 27)

Nous verrons ci-dessous qu'il n'y a pas, dans toute la littérature française, un écrivain qui ait plus fait l'apologie du progrès que Victor Hugo.

\section{Le Prophète du progrès}

Pierre Albouy, d'instinct, avait deviné que la perception que les lecteurs allaient avoir du rapport de Hugo à la science serait forcément erronée. Nous le répétons : la périodisation en littérature et les procédés simplificateurs des anthologies et des manuels scolaires, en recourant le plus souvent aux techniques de l'histoire littéraire, étiquettent les auteurs et les clouent dans des catégories préfinies desquelles ils ressortent très rarement. Albouy rectifie :

Loin d'être l'ennemi de la science, Hugo, au contraire, voudrait la voir plus hardie. Peut-être, direz-vous, manque-t-il un peu d'esprit critique ? Mais c'est un poète, un homme d'imagination. L'essentiel, c'est qu'à propos de la science on retrouve son attitude d'optimisme, son esprit de conquête et de progrès, sa confiance dans l'homme. (ALBOUY, 1976, p. 105)

Les appels du poète au progrès sont si poignants que Pierre Brunel 
parlera d'une " idéologie du progrès ", qualifiant ensuite Hugo de "l'être le plus convaincu par l'idéologie du Progrès" (BRUNEL, 2000, p. 115). La foi du poète à l'endroit de la science est si intense qu'elle semble souvent un zèle de néophyte. La réputation d'athée qui lui est faite par certains trouve peutêtre son explication à ce niveau. L'expression de " religion du progrès " est courante dans son ouvre. Dans Les Misérables, il dira des jeunes étudiants, membres du club de L'ABC : "Tous ces jeunes gens, si divers, et dont, en somme, il ne faut parler que sérieusement, avaient une même religion : le Progrès » (HUGO, 1962a, p. 601).

On a pris l'habitude d'oublier que l'auteur des Contemplations avait le profil idéal pour réussir l'Ecole Polytechnique (DECAUX, 2001). Avec Eugène, son frère aîné, son père l'emmène à la pension Cordier pour justement en préparer le concours. Son aisance dans le maniement de la terminologie scientifique est notoire. On pourra à ce titre constater que l'essentiel des éditions des Travailleurs de la mer (HUGO, 1962b) s'accompagnent d'un glossaire pour rendre plus accessibles les références aux espèces et courants marins, le lexique technique de la marine marchande, la terminologie météorologique, etc. Hugo aurait, pour se familiariser avec les diverses branches de l'océanographie, exploité une bibliographie équivalente à celle d'une thèse d'État (EIGELDINGER, 1980, p. V). En outre, dès 1852 , le poète - qui a fui la France à bord d'un train, au soir du 2 décembre 1851 - est profondément convaincu que la science décuple les moyens de l'homme (à la différence de la quasi-totalité de ses pairs d'une Académie encore largement assise sur les certitudes de l'Ancien Régime). Par conséquent

Il applaudit aux applications des découvertes de Watt, Stephenson, Volta, Ampère, Lagrange, La Harpe, Claude Bernard, Charcot. La distance et le temps son raccourcis grâce au chemin de fer, au steamer, à l'électricité et au télégraphe. Plus tard, il encouragea Nadar dans l'expérimentation de son ballon dirigeable. [...] Dans ce type d'analyse, il se révèle plus matérialiste qu'il ne croit. (BOUGNOUX, 2002, p. 32)

Pour Hugo, la grandeur de la science réside d'abord dans le fait qu'elle sacre l'homme co-créateur, voleur du feu sacré; ensuite parce qu'elle le libère de ses vieilles peurs paniques, comme le précise dans Les Misérables cette harangue de Enjolras, le chef des étudiants insurgés :

Jadis les premières races humaines voyaient avec terreur passer devant leurs yeux l'hydre qui soufflait sur les eaux, le dragon qui vomissait du feu, le griffon qui était le monstre de l'air [...] Nous avons dompté l'hydre, et elle s'appelle le steamer ; nous avons dompté le dragon, et il s'appelle 
la locomotive ; nous sommes sur le point de dompter le griffon, nous le tenons déjà, et il s'appelle le ballon. Le jour où cette œuvre prométhéenne sera terminée [...] l'homme aura définitivement attelé à sa volonté la triple Chimère antique, l'hydre, le dragon et le griffon! (HUGO 1962a, p. 779)

Cette métaphore de l'homme est obsédante dans les récits hugoliens : défavorisé physiquement, son intelligence le propulse cependant au sommet de la nature. Dans Quatrevingt-Treize, lorsque finalement un marin réussit à reprendre le contrôle d'un canon mal arrimé (qui écrase tout sur son passage), le narrateur conclut : "C'était fini. L'homme avait vaincu. La fourmi avait eu raison du mastodonte ; le pygmée avait fait le tonnerre prisonnier " (HUGO, 1962c, p. 1302).

En outre, le Progrès est le grand héritage de la rhétorique révolutionnaire ; il est le nouveau point de ralliement des grands esprits dont Hugo est convaincu de la mission prométhéenne, et, par voie de fait, de la dimension titanesque. Parlant desdits esprits dans Les Misérables, son narrateur commente : "L'immense avance de l'humanité vers la lumière leur est due "(HUGO, 1962a, p. 714). C'est pratiquement sous les traits de Mirabeau qu'Enjolras, aux étudiants insurgés, lance cette prédiction : "Citoyens, le dix-neuvième siècle est grand, mais le vingtième siècle sera heureux » (HUGO, 1962a, p. 779). Cette prophétie est à mettre au crédit de l'absolue confiance que l'écrivain a alors à l'endroit de ce qui, pas à pas, s'édifie sous ses yeux, partout en Europe comme aux Etats-Unis : le grand avènement de la machine. Car pour Hugo progrès signifie d'abord amélioration matérielle. On peut lire à ce titre l'étude que Myriam Roman a consacrée à l'idéologie du progrès chez l'écrivain (ROMAN, 2000). Le premier axe fait référence à la conception multidimensionnelle du progrès dans l'œuvre hugolienne, avec pour première acception du mot le développement scientifique et technique dont il fait l'éloge au point de s'en signaler comme l'un des porte-étendards. C'est donc tout naturellement qu' " en 1867, Hugo fait partie des cent vingt-cinq écrivains ou techniciens qui collaborent au Paris-Guide, composé pour l'Exposition Universelle de 1867, vitrine triomphale des progrès de la science» (ROMAN, 2000, p. 76). En outre, le Progrès - un " de ces mots que Hugo écrit avec une majuscule pour souligner leurs nature idéelle " (ROMAN, 2000, p. 76) - est saisi dans son aspect moral et politique, mais il est surtout perçu comme fin ultime de l'Histoire :

L'idée de progrès est perceptible à différents niveaux, depuis les progrès dans les sciences et les techniques, jusqu'au progrès moral, individuel (l'éthique) et collectif (la Révolution). L'œuvre de l'exil met en valeur ces différents 
aspects. La connaissance scientifique fait l'objet d'un livre entier de William Shakespeare [...] Les Travailleurs de la mer racontent l'introduction du bateau à vapeur dans les îles anglo-normandes [...] dans Les Misérables, Monsieur Madeleine fait sa fortune et celle de Montreuil-sur-Mer grâce à une invention technique. (ROMAN, 2000, p. 79)

D'autre part, dans les divers genres où s'essaie l'auteur, progrès rime tout aussi bien, avec amélioration morale, conséquence obvie du progrès matériel, pas de plus dans l'éternelle marche de l'humanité vers l'Idéal. Si donc l'écrivain s'est parfois montré fervent partisan de la colonisation française en Afrique $^{2}$, ce n'est point, au fond, par la croyance à une quelconque supériorité raciale qui accorderait un tel privilège aux "dominants". En réalité Hugo a naïvement cru à la mission civilisatrice d'une France portant le flambeau de ses sciences partout où persistent encore les ténèbres. Le progrès moral résume ainsi tous les autres symboles du concept, étant le but suprême de l'homme ici-bas car, comme le précise l'auteur dans Le Tas de pierres, « le but humain n'est pas le but animal ( HUGO, 1962d, p. 1411).

Néanmoins, de tout ceci, il ne faudrait pas conclure que si Hugo se veut le chantre du progrès, il n'est nullement un héraut aveugle. Il aura assez vécu pour se rendre compte (avec le privilège du témoin oculaire) que la Révolution industrielle n'a pas bâti l'Eldorado social que son optimisme y plaçait. Le poète (qui voyait dans la machine le nouveau Pégase chevauché par l'homme) découvre atterré, par ce que Marx allait appeler " aliénation " (MARX, 1993, p. 100), la créature prenant la place de son créateur. Ainsi, quand il reparle du progrès, son enthousiasme trahi s'en fait le lucide critique puis, graduellement, l'impitoyable contempteur. Pierre Albouy a remarqué cette évolution dans la pensée de l'écrivain :

La critique de ces dernières années a montré combien la notion de progrès constituait une idée à la fois centrale et aporétique dans l'œuvre de Victor Hugo, en particulier à partir de l'exil [...] Pourtant, le progrès hugolien n'a rien d'un optimisme béat, et s'offre au contraire dans le paradoxe et l'oxymore. (ALBOUY, 1976, p. 75)

C'est pourquoi, même si cinq parties structurent l'article de Myriam Roman cité ci-dessus, deux axes majeurs en constituent les piliers : «L'Optimisme hugolien : Hugo, chantre du Progrès » et « Le pessimisme hugolien : le progrès, «lumineux désastre »".

${ }^{2}$ Propos relevé d'un discours du poète (prononcé à propos du projet colonial français en Afrique lors d'un banquet donné en 1879) dans lequel il est martelé la responsabilité des « races supérieures » devant porter les Lumières aux « races inférieures ». Ce discours a été publié dans le Journal officiel de la République française du 28 juillet 1885. 


\section{Le grand déçu du progrès}

Hugo a pendant longtemps envisagé la nécessité d'accepter le contrepoids du progrès qui, parce qu'il n'emprunte pas toujours des sentiers fleuris, est souvent déroutant dans sa démarche ; les révolutions en font foi. L'œuvre, une fois encore, se fait le miroir réflexif de cette philosophie. Dans Quatrevingt-treize, la guerre civile postrévolutionnaire n'est pas totalement à déplorer puisqu' "en démontrant la nécessité de trouer dans tous les sens la vieille ombre bretonne et de percer cette broussaille de toutes les flèches de la lumière à la fois, la Vendée a servi le progrès. Les catastrophes ont une sombre façon d'arranger les choses" (HUGO, 1962c, p. 1351). Les Misérables renferme quasiment la même justification de la guerre, celle de Waterloo qui, malgré le sinistre qu'elle cause sur le plan humain, a signé la fin du dernier bastion de la féodalité en Europe. Ensuite, la guerre est perçue comme cet alambic duquel sortent sublimées des têtes-brûlées. Un commentaire du narrateur des Misérables est une postulation du malnécessaire des grandes mêlées :

Voulez-vous vous rendre compte de ce que c'est que la révolution, appelez-la
Progrès ; et voulez-vous vous rendre compte de ce que c'est que le progrès,
appelez-le Demain. Demain fait irrésistiblement son œuvre, et il la fait dès
aujourd'hui. Il arrive toujours à son but, étrangement. Il emploie Wellington
à faire de Foy, qui n'était qu'un soldat, un orateur. Foy tombe à Hougomont
et se relève à la tribune. Ainsi procède le progrès. Pas de mauvais outil pour
cet ouvrier-là. (HUGO, 1962a, p. 1358)

L'idée est plus tard allégorisée à travers la personne du capitaine Gauvain. Ses opérations militaires sont aussi bien des opérations chirurgicales. Son père spirituel "Cimourdin se figurait Gauvain écrasant du pied les ténèbres cuirassé de lumière, avec une lueur de météore au front, ouvrant les grandes ailes idéales de la justice, de la raison et du progrès, et une épée à la main ; ange, mais exterminateur » (HUGO, 1962c, p. 1358, [nous soulignons, MF]).

L'écrivain emploie ce procédé antithétique (sa marque de fabrique ${ }^{3}$ ) structuré autour du " mais » pour rendre compte du travail de la Convention :

Orateurs, prophètes, hommes-colosses comme Danton, hommes-enfants comme Cloots, gladiateurs et philosophes, tous allaient au même but, le

\footnotetext{
${ }^{3}$ "L'univers hugolien - renseigne Pierre Albouy - est toujours antithétique ; tout nadir y suppose un zénith» (ALBOUY, 1976, p. 190).
} 
progrès. En même temps qu'elle dégageait de la révolution, cette assemblée produisait de la civilisation. Fournaise, mais forge. Dans cette cuve où bouillonnait la terreur, le progrès fermentait. (HUGO, 1962c, p. 1336)

Cette métaphore obsédante qu'il donne du progrès est une constante dans l'œuvre du Hugo des années d'exil. Elle pourrait s'expliquer par l'ardent désir qui l'habite alors d'en finir avec la monarchie renaissant en France avec Louis Napoléon, fût-ce par la lutte armée. Des vers dans Les Contemplations rappellent cette obsession ressassée dans un mémorable chiasme : "Les Révolutions, qui viennent tout venger, / Font un bien éternel dans leur mal passager »(HUGO, 1962g, p. 435). Lorsque six années plus tard il publie Les Misérables, la même image ressurgit, cette fois-ci en prose. C'est ici sa justification de la déliquescence des religions au siècle de la Révolution industrielle :

Au dix-neuvième siècle, l'idée religieuse subit une crise. On désapprend de certaines choses, et l'on fait bien, pourvu qu'en désapprenant ceci, on apprenne cela. Pas de vide dans le cœur humain. De certaines démolitions se font, et il est bon qu'elles se fassent, mais à la condition d'être suivies de reconstructions. (HUGO, 1962a, p. 552)

Cette conception particulière du progrès est en soi un aspect autobiographique dans l'œuvre de Hugo. Lors des échauffourées de 1851, n’a-t-il pas écrit: «On se souvient des morts qui ont déterminé des indignations et des soulèvements. On n'a plus que cette ambition : être un cadavre utile " ? (DECAUX, 2001, p. 771).

Mais, progressivement, la rupture s'opère. Avec l'âge on assiste à l'ébranlement de certains principes. Le patriarche exilé se rend de plus en plus compte que destruction n'est pas toujours suivie de reconstruction dans cette Europe en phase accélérée de mécanisation. Sa correspondance et les fréquentes demandes d'intervention qu'il reçoit lui font très vite comprendre qu'il sera confronté, dans son siècle, à plus d'une affaire Calas. La Révolution industrielle est au fond la Terre promise d'une très faible minorité sociale, ces happy-few auxquels il jette la pierre dans Les Misérables, ces «spectateurs tranquilles de la douleur [...] déterminés à être heureux jusqu'à épuisement du rayonnement des astres et du chant des oiseaux " (HUGO, 1962a, p. 863).

En fait, Hugo est un grand optimiste, très souvent déçu, ses rêves prenant des formes monstrueuses. Le Progrès qu'il attendait du régime de Napoléon III - dont il a beaucoup aidé à faciliter l'élection en 1848 - ne s'est pas réalisé : la Révolution industrielle réveillerait-elle ses vieux monstres 
qui le hantent depuis le coup d'Etat du 2 décembre? Un critique ne manque pas de faire la corrélation avec le métaphorique naufrage de la Durande, ce révolutionnaire bateau à vapeur dans Lfes Travailleurs de la mer qui a osé remplacer les classiques mais incertaines voiles du progrès par de sûrs moteurs à pistons : "La destruction de la Durande dans Les Travailleurs de la mer, lit-on chez Albouy, peut être lue comme une fable politique de la Révolution naufragée par le coup d'Etat du 2 décembre [...] L'histoire du XIX siècle offre plutôt l'histoire de révolutions avortées " (ALBOUY, 1976, p. 82). Ainsi, la Révolution industrielle a fait faillite en ce qu'elle n'a pas mis un terme à la misère sociale. Tout le contraire de la Terre promise qu'y voyaient ses plus ardents défenseurs, l'avènement de la machine va mettre en exergue l'indicible misère sociale caractérisant les couches sociales les plus fragiles. La découverte de cet insurmontable paradoxe s'est faite sur la base d'études scientifiques trente ans avant Les Misérables et un demi-siècle avant les macabres descriptions des conditions de vie prolétariennes dans Germinal. En effet

Dès 1834, l'économiste Villeneuve-Bargemont affirme que l'indigence sous le nom, nouveau et tristement énergique, de paupérisme envahit des classes entières de la population, tend à s'accroître progressivement en raison même de la production industrielle. Elle n'est plus un accident, mais la condition forcée d'une grande partie des membres de la société. (PEÑA-RUIZ ; SCOT, 2002, p. 315)

A partir des Contemplations se sent l'insurmontable crise de conscience qui habite l'écrivain et qui allait, six années plus tard, exploser à travers cette grande plaidoirie sociale que sont Les Misérables. D'autre part, l'auteur qui se sent déjà " au bord de l'Infini " ne peut décidément pas oublier une malédiction qu'il a lui-même jetée un certain soir de 1840 dans Les Rayons et les ombres: "Malheur à qui prend ses sandales / Quand les haines et les scandales / Tourmentent le peuple agité ! / Honte au penseur qui se mutile / Et s'en va, chanteur inutile, / Par la porte de la cité ! " (HUGO, 1962h, p. 45).

Qu'on n'oublie pas que Hugo est quasiment devenu prophète d'un peuple au secours duquel il se dévoue. Il sent l'impérieux devoir d'assumer la dimension mythique désormais sienne. Bref, il se sent investi d'une mission. Héros déclaré d'un peuple, Victor Hugo, comme tous ceux que le destin ou leurs efforts propres placent sur un tel piédestal, doit se trouver une cause. Ce sera celle des enfants.

Ainsi, bien avant Le Capital de Marx, le voici qui lithographie les obscurs contours d'un progrès industriel devenu Léviathan pour un peuple 
spolié. Dans "Melancholia ", à travers des vers d'une matité saisissante, il devance l'ONU de plus d'un siècle dans l'indigné « carton rouge au travail des enfants" :

Où vont tous ces enfants dont pas un seul ne rit?

Ces doux êtres pensifs, que la fièvre maigrit ?

Ces filles de huit ans qu'on voit cheminer seules?

Ils s'en vont travailler quinze heures sous des meules;

Ils vont, de l'aube au soir, faire éternellement

Dans la même prison le même mouvement.

Accroupis sous les dents d'une machine sombre,

Monstre hideux qui mâche on ne sait quoi dans l'ombre,

Innocents dans un bagne, anges dans un enfer,

Ils travaillent. Tout est d'airain, tout est de fer.

[...] Progrès dont on demande : "Où va-t-il ? que veut-il ? "

Qui brise la jeunesse en fleur! qui donne, en somme,

Une âme à la machine et la retire à l'homme ! (HUGO, 1962g, p. 403).

En un mot, le poète se radicalise dans l'expression de son désenchantement au sujet de la science. Il ne peut plus accepter l'idée d'une quelconque lumière issue de la foudre. Un siècle avant Saint-John Perse, il réalise que "c'est de l'homme qu'il s'agit, dans sa présence humaine " (PERSE, 1972, p. 224). Tout progrès se faisant donc au détriment du bonheur du plus grand nombre est alors perçu pour un leurre. Selon Pierre Albouy, Hugo n'avait pas aveuglément fait appel à la science de manière unilatérale, mais

À une science démocratique, au service de l'homme et de sa liberté, l'exigence d'une science qui se refuserait à l'indifférence en matière d'humanité, quand il est question, pour l'homme, de vie ou de mort ; une science qui se voudrait en accord avec la morale, et non pas une sorte de monstre aveugle et terrible, une sorte de super-fléau de la nature, utilisé par des hommes contre les hommes, une science, oui, qui aurait, sinon de l'âme, du moins du cœur. (ALBOUY, 1976, p. 103)

Cette évolution de la pensée du poète à l'égard de la science, Émilie Tiollier-Aqajani l'a aussi bien remarquée dans les recueils de poésie comme La Légende des siècles. Elle y observe qu'" en vérité Hugo n'a de cesse de fustiger la pensée d'Auguste Comte, chantre du positivisme, qui domina toute la seconde moitié du XIX ${ }^{\mathrm{e}}$ alors en plein développement industriel » (TIOLLIER-AQAJANI, 2013, p. 159). Désormais le penseur ne se sent plus la force de cautionner l'idée du progrès " ange mais aussi exterminateur "; il en a la nausée. Il déclare, à ce propos, dans Littérature et philosophie mêlées: 
Pour beaucoup de raisonneurs à froid qui font après coup la théorie de la Terreur, 93 a été une amputation brutale, mais nécessaire. Robespierre est un Dupuytren politique. Ce que nous appelons la guillotine n'est qu'un bistouri. C'est possible. Mais il faut désormais que les maux de la société soient traités non par le bistouri, mais par la lente et graduelle purification du sang, par la résorption prudente des humeurs extravasées, par la saine alimentation, par l'exercice des forces et des facultés, par le bon régime. $\mathrm{Ne}$ nous adressons plus au chirurgien, mais au médecin. (HUGO, 1962e, p. 1180)

Cette palinodie trouve également une page dans Les Misérables, c'està-dire le roman miroir de l'extrême misère sociale qui gangrène certaines couches humaines du siècle. Hugo la signe par la dernière harangue d'Enjolras (celui-là même qui défendait l'idée du progrès violent) :

La nécessité est un monstre du vieux monde ; la nécessité s’appelle Fatalité. Or, la loi du progrès, c'est que les monstres disparaissent devant les anges, et que la Fatalité s'évanouisse devant la fraternité. C'est un mauvais moment pour prononcer le mot amour. N'importe, je le prononce, et je le glorifie. Amour, tu as l'avenir. Mort, je me sers de toi, mais je te hais. Citoyens, il n'y aura dans l'avenir ni ténèbres, ni coups de foudre, ni ignorance féroce, ni talion sanglant. Comme il n'y aura plus de Satan, il n'y aura plus de Michel. Dans l'avenir personne ne tuera personne, la terre rayonnera, le genre humain aimera barricade. (HUGO, 1962a, p. 754)

\section{Conclusion}

Lorsqu'on revient sur les premiers pas de Victor Hugo, on s'étonnera sans doute de le voir, à un moment de sa vie, se faire l'un des plus célèbres apologistes du progrès scientifique. Très doué en mathématiques, le jeune Hugo de la pension Cordier n'est pas cependant particulièrement attiré par les sujets de science. Quand il n'arpente pas le jardin des Feuillantines en quête de futurs motifs poétiques, on le retrouve aux jeux floraux à faire couronner des Odes dont la plupart ont été signées de la main d'un extraordinaire prodige de dix ans. On sait qu'il refusera de se présenter au concours de l'école polytechnique malgré les ordres de son père et toutes les dispositions qui lui y préparaient une belle carrière. Cependant, le rapport de Hugo à la science va radicalement changer quand il en découvre les résultats presque miraculeux. Pour beaucoup de ses biographes, cette découverte des trésors de la science date de son exil qui lui permet d'admirer, sur la route de Bruxelles, la locomotive puis le bateau à vapeur en Angleterre. Nous pensons à notre niveau que la fascination de l'auteur devant la science remonte bien plus loin. Déjà, dès les années 1830 - période faste du drame 
romantique dont les mises en scène sophistiquées requièrent les dernières avancées techniques, la science lui fait concevoir que " ceci tuera cela".

En effet, dans son roman Notre-Dame de Paris, le docteur Claude Frollo, après avoir prouvé à son public comment l'architecture fut le "s seul livre des peuples " pendant tout le temps pré-gutemberguien, brandit soudain un ouvrage imprimé d'une main, et de l'autre indexant la lourde cathédrale Notre-Dame se découpant à l'horizon, s'écria : "Hélas ! Ceci tuera cela "(HUGO, 1962i, p. 98). Pour ce savant, la pensée se gravant beaucoup plus facilement sur le papier que sur la pierre, et s'y découvrant plus commodément, par cette immuable loi faisant que " les petites choses viennent à bout des grandes " (HUGO, 1962i, p. 98), l'invention de l'imprimerie a sonné la fin de la pensée dans l'édifice. Compris ainsi, il est une logique inaltérable qui exige pour chaque nouveau progrès de nécessairement faire table rase du tremplin de l'ancien. La conscience de l'auteur se fait à la loi d'un progrès démolisseur-bâtisseur. À partir de ce moment, l'œuvre intègre la recherche scientifique dans son actualité la plus immédiate (comme on peut le voir de nos jours avec l'auteur de romans policiers Dan Brown) : le titre d'un livre de David Charles en dit long : La Pensée technique dans l'œuvre de Victor Hugo (2003). Plus que jamais amoureux du peuple qui a motivé son exil, son œil visionnaire lui déroule très vite les plans d'un avenir de l'humanité où la science conduirait au soulagement des plus faibles. Dès lors Hugo se lance en campagne ouverte pour la science, sans demi-mesure, comme il s'y était déjà pris avec ses deux fils pour faire élire à la présidence Louis-Napoléon. Mais là encore la déception est grande. Ce désenchantement est à mettre en étroite relation avec l'extrême misère des classes ouvrières dans cette seconde moitié du $\mathrm{XIX}^{\mathrm{e}}$ siècle. Bien avant Zola, Victor Hugo se rend compte que la science, à l'instar de la locomotive qui avale des forêts pour avancer, broie des masses d'hommes pour réaliser l'eldorado de quelques-uns. Loin donc de son obsession des structures antithétiques, sa palinodie à l'endroit de la science s'explique surtout par la révolution ratée à laquelle le plus grand progrès de l'humanité aboutit, c'est-à-dire la Révolution industrielle.

\section{Bibliographie}

\section{Sources}

HUGO, Victor. Euvres romanesques complètes : réunies et présentées par Francis Bouvet. t. 1. Paris: Jean-Jacques Pauvert, 1962. 


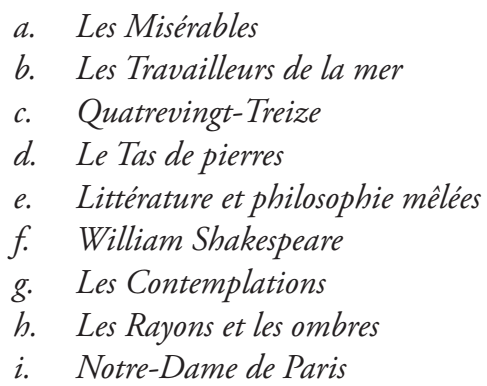

\section{Ouvrages critiques et théoriques}

ALBOUY, Pierre. Mythographies. Paris : José Corti, 1976.

BAFARO, Georges. Le roman réaliste et naturaliste. Paris : Edition Marketing, 1995.

BOUGNOUX, Daniel. Hugo/Aragon : deux œuvres en miroir. Le Magazine littéraire, Victor Hugo, deux siècles de légende, n. 405, p. $32-$ 56, 2002.

BRUNEL, Pierre. Monsieur Victor Hugo. Tournai : Vuibert, 2000. CHARLES, David. La Pensée technique dans l'œuvre de Victor Hugo. Paris : Presses Universitaires de France, 2003.

DECAUX, Alain. Victor Hugo. Paris : Perrin, 2001.

DUPRONT, Alphonse. Qu'est-ce que « Les Lumières ». Paris : Gallimard, 1996.

EIGELDINGER, Marc. Introduction aux Travailleurs de la mer de Victor Hugo. Paris : Garnier-Flammarion, 1980.

JAUSS, Hans Robert. Pour une esthétique de la réception. Paris : Gallimard, 2015.

MARX, Karl. Le Capital. t. I. Paris : Presses Universitaires de France, 1993. MAUROIS, André. Lettre ouverte à un jeune homme. Paris : Albin Michel, 1966.

PENAA-RUIZ, Henri; SCOT, Jean-Paul. Un poète en politique: Les combats de Victor Hugo. Paris : Flammarion, 2002.

PERSE, Saint-John. Guvres poétiques complètes. Vents. Paris : Gallimard, 1972.

ROMAN, Myriam. Ce cri que nous jetons souvent. Romantisme, L'idée de progrès, n. 108, p. 75-90, 2000.

TIOLLIER-AQAJANI, Émilie. Lécriture du Moi dans La Légende des 
siècles de Victor Hugo : entre condensation et dilatation. Les Cahiers du GRELCEF, n. 4, p. 155-168, 2013. 